\title{
Consenso Brasileiro de Monitorização e Suporte Hemodinâmico - Parte IV: Monitorização da Perfusão Tecidual
}

\author{
Brazilian Consensus of Monitoring and Hemodynamic \\ Support - Part IV: Tissue Perfusion Evaluation
}

\author{
Álvaro Réa-Neto, Ederlon Rezende, Ciro Leite Mendes, Cid Marcos David, \\ Fernando Suparregui Dias, Guilherme Schettino, Suzana Margareth Ajeje Lobo pelo \\ Painel de Especialistas do Consenso Brasileiro de Monitorização e Suporte Hemodinâmico.
}

\section{RESUMO}

JUSTIFICATIVA E OBJETIVOS: A manutenção da perfusão e da oferta de oxigênio às células, para satisfazer o seu metabolismo, é a principal função do sistema cardiorrespiratório. Fisiologicamente, tanto a perfusão quanto à oferta de oxigênio aos tecidos é controlada pela taxa metabólica celular. Os pacientes criticamente doentes estão com alto risco de hipoperfusão tecidual e esta está diretamente relacionada com lesão orgânica e disfunção de múltiplos órgãos. Portanto, a monitorização da perfusão sistêmica é parte integrante e indissociável da avaliação hemodinâmica de qualquer paciente criticamente doente e está indicada em todos estes pacientes. $\mathrm{O}$ objetivo foi determinar a utilidade clínica das diferentes formas de monitorização da perfusão à beira do leito.

MÉTODO: O processo de desenvolvimento de recomendações utilizou o método Delphi modificado para criar e quantificar o Consenso entre os participantes. A AMIB determinou um coordenador para o Consenso, o qual escolheu seis especialistas para comporem o Comitê Consultivo. Outros 18 peritos de diferentes regiões do país foram selecionados para completar o painel de 25 especialistas, médicos e enfermeiros. Um levantamento bibliográfico na MedLine de artigos na língua inglesa foi realizado no período de 1966 a 2004. RESULTADOS: Foram apresentadas recomendações acerca da utilidade da monitorização clínica da perfusão tecidual, gradiente de temperatura e medida da oxigenação transcutânea, lactato sérico, excesso de base, $\mathrm{SvO}_{2} / \mathrm{SvcO}_{2}$, tonometria gasosa gastrintestinal e capnometria gasosa sublingual, gradiente veno-arterial de $\mathrm{CO}_{2}$ e Orthogonal Polarization Spectral (OPS).

CONCLUSÕES: A compensação hemodinâmica de um paciente criticamente doente não se completa sem que a perfusão tecidual sistêmica seja corrigida. Vá- rios métodos de monitorização estão disponíveis e são úteis na prática clínica, entretanto, nenhum deles tem características de acurácia e efetividades suficientes para serem utilizados independentemente do contexto clínico.

Unitermos: Consenso, Monitorização Hemodinâmica, Perfusão Tecidual, Recomendação.

\section{SUMMARY}

BACKGROUND AND OBJECTIVES: The main cardiovascular function is to maintain the adequate perfusion e oxygen delivery to the cells. Physiologically, this is controlled by the cellular metabolic rate. The critically ill patients are in high danger of tissue hipoperfusion and this is directly related to cellular injury and organ dysfunction. Therefore, the tissue perfusion monitoring makes part and is indissociated of hemodynamic evaluation of the critically ill patient and is indicated to all this patients. The objective was to define recommendations about clinical utility of different tolls to bedside perfusion monitoring.

METHODS: Modified Delphi methodology was used to create and quantify the consensus between the participants. AMIB indicated a coordinator who invited more six experts in the area of monitoring and hemodynamic support to constitute the Consensus Advisory Board. Twenty five physician and two nurses selected from different regions of the country completed the expert panel, which reviewed the pertinent bibliography listed at the MedLine in the period from 1996 to 2004.

RESULTS: Recommendations were done about the utility of clinical monitoring of tissue perfusion, temperature gradient and transcutaneous oxygen monitoring, serum lactate, base excess, $\mathrm{SvO}^{2}$ and $\mathrm{ScvO}^{2}$, gastric and sublingual capnometry, $\mathrm{CO}^{2}$ venous-arterial gradient and Orthogonal Polarization Spectral (OPS). 
CONCLUSIONS: The homodynamic compensation of a critically ill patient isn't complete unless the tissue perfusion is corrected. Many different methods of monitoring is available and are useful in clinical practice, however, none has accuracy and effectiveness characteristics to be used independently of clinical context.

Key Words: Consensus, Hemodynamic Monitoring, Recommendation, Tissue Perfusion.

\section{INTRODUÇÃO}

A manutenção da perfusão e da oferta de oxigênio às células, para satisfazer o seu metabolismo, é a principal função do sistema cardiorrespiratório. Fisiologicamente, tanto a perfusão quanto à oferta de oxigênio aos tecidos é controlada pela taxa metabólica celular. Os pacientes criticamente doentes estão em alto risco de hipoperfusão tecidual, a qual está diretamente relacionada com lesão orgânica e disfunção de múltiplos órgãos. Portanto, a monitorização da perfusão sistêmica é parte integrante e indissociável da avaliação hemodinâmica de todo paciente crítico e está indicada em todos. A complexidade da monitorização é que deve ser individualizada. Entretanto, todos os pacientes críticos, compensados ou não, devem ter a monitorização da perfusão sistêmica estimada ou aferida, concomitantemente à monitorização de outros parâmetros hemodinâmicos.

Muito freqüentemente, as manifestações relacionadas a distúrbios da perfusão tecidual ocorrem antes de outras manifestações hemodinâmicas (hipotensão arterial, diminuição da PVC, etc.) e normalizam-se posteriormente. Por isso, elas são sinais precoces de alterações hemodinâmicas e a compensação clínica de um paciente crítico só se completa com a normalização da perfusão tecidual.

Nenhuma forma de avaliar a perfusão tecidual é 100\% sensível ou específica, então qualquer avaliação deve ser inserida no contexto clínico do paciente.

\section{QUESTÃO: A MONITORIZAÇÃO CLÍNICA DA PER- FUSÃO TECIDUAL TEM UTILIDADE NA PRÁTICA DE BEIRA DO LEITO?}

Recomendação: Sim. A monitorização clínica da perfusão tecidual envolve um conjunto de sinais e sintomas inespecíficos e de sensibilidade limitada, mas de fácil e rápida interpretação que envolve custos e riscos mínimos. É útil na avaliação inicial de pacientes críticos e deve ser realizada rotineiramente. No entanto, deve-se conhecer as limitações dessas informações e a necessidade de integrá-las ao contexto clínico do paciente ,para que possam ser apropriadamente utilizadas no processo de decisão de monitorização mais avançada e terapêutica. É operador dependente e alguns dos sinais clínicos podem aparecer tardiamente quando importante hipoperfusão já ocorreu, como é o caso da hipotensão arterial.

Ela é especialmente útil para descrever uma condição clínica basal com o intuito de avaliar os resultados dos outros índices de perfusão/oxigenação descritos a seguir. Como nenhum método de avaliação da perfusão é definitivo, qualquer resultado deve sempre ser incluído na perspectiva descrita pela monitorização clínica da perfusão tecidual.

Grau D

Racional: O primeiro passo para o sucesso no tratamento de estados de choque é o reconhecimento precoce da presença de hipoperfusão. Não existe um sinal, sintoma ou exame laboratorial que diagnostique choque isoladamente ${ }^{1}$. A hipotensão arterial é um indicador tardio de hipoperfusão e lesão significativa pode ocorrer antes de seu aparecimento. Quase 30\% do volume circulante podem ser perdidos antes do aparecimento de hipotensão arterial significativa ${ }^{2}$. Portanto, o choque precisa ser reconhecido antes que a hipotensão se instale. Um dos sinais mais precoce a ser observado é o estreitamento da pressão de pulso secundária a uma pequena diminuição da pressão sistólica e a elevação da pressão diastólica que ocorre devido ao aumento de catecolaminas circulantes. É, portanto, imperativo estar alerta para um grupo de sinais e sintomas inespecíficos sugestivos de perfusão inadequada que, no contexto clinico apropriado, possibilita um diagnóstico precoce de choque, mesmo na ausência de hipotensão arterial significativa. A taquicardia ocorre após perda de aproximadamente $15 \%$ do volume circulante, mas é importante lembrar que essa resposta pode estar abolida em pacientes em uso de beta- bloqueadores ou portadores de marca-passo. A presença de livedo e extremidades frias associadas ao aumento do tempo de enchimento capilar sinaliza vasoconstrição reflexa em resposta a hipovolemia, mas pode também ocorrer em situações de doença vascular periférica. Alterações dos níveis de consciência aparecem precocemente quando existe hipoperfusão, mas podem representar também efeitos colaterais de fármacos ou um evento envolvendo o sistema nervoso central. Oligúria e elevação de escórias nitrogenadas são importantes, não somente no diagnóstico, como no manuseio dos estados de choque, 
mas podem estar também presentes devido ao uso de fármacos nefrotóxicos e outras causas de insuficiência renal, não necessariamente ligadas à presença de hipoperfusão. Sede e urina concentrada antecedem a elevação de escórias nitrogenadas e sinalizam precocemente estados de hipovolemia. A presença de taquipnéia, em especial, na ausência de achados anormais à ausculta pulmonar ou anormalidade radiológica pode ser sugestiva da presença de acidose metabólica necessitando de compensação respiratória, sendo também mais um sinal inespecífico que pode auxiliar no diagnóstico precoce dos estados de hipoperfusão ${ }^{3-8}$.

\section{QUESTÃO: A MONITORIZAÇÃO DO GRADIENTE DE TEMPERATURA E A MEDIDA DA OXIGENAÇÃO TRANSCUTÂNEA TEM UTILIDADE NA PRÁTICA CLÍNICA?}

Recomendação: Sim, com limitações. Esses métodos baseiam-se no fato de que a vasoconstrição cutânea é um sinal precoce de hipoperfusão. A medida do gradiente de temperatura consiste na aferição da temperatura em 2 pontos distintos: um periférico (p. ex.: dedos dos pés) e um central (p. ex.: esofagiano). Visto que a vasoconstrição da pele reduz o metabolismo periférico, a diferença entre as temperaturas central e da pele pode aumentar. A medida da oxigenação transcutânea $\left(\mathrm{PtcO}_{2}\right)$ é realizada com eletrodo de fixação minimamente invasivo na pele. As tensões de oxigênio subcutâneo e transcutâneo refletem índices confiáveis da $\mathrm{PaO}_{2}$ apenas em variadas situações de normovolemia.

Grau B2

Racional: A diferença entre a temperatura central e periférica tem sido usada para diagnosticar e tratar pacientes com anormalidades de fluxo sanguíneo global. Um gradiente de temperatura central para periférica de $3^{\circ} \mathrm{C}$ a $7^{\circ} \mathrm{C}$ ocorre tão logo a hemodinâmica do paciente tenha sido otimizada. Valores maiores que $7^{\circ} \mathrm{C}$ podem ser indicativos de hipoperfusão ${ }^{9}$. Medidas do gradiente de temperatura podem ser úteis para avaliar de forma não-invasiva o fluxo sanguíneo periférico e para sinalizar a gravidade do estado de choque, auxiliando no diagnóstico de hipoperfusão, mesmo na presença de valores normais de índice cardíaco ${ }^{7-10}$. No choque cardiogênico, correlaciona-se melhor com o índice cardíaco e variáveis de transporte de oxigênio do que a $\mathrm{PtcO}_{2}{ }^{8}$. É importante, no entanto, reconhecer que esse gradiente tem valor limitado em pacientes com doença arterial periférica, estado de hipotermia e choque com vasodilatação. Pode também sinalizar vasoconstrição periférica excessiva por fármacos de ação vasoconstritoras ${ }^{11,12}$. A $\mathrm{PtcO}_{2}$ reflete a $\mathrm{PO}_{2}$ nos tecidos periféricos e varia com as alterações cardiopulmonares que afetam a oxigenação tissular. Em estados de hipoperfusão, a $\mathrm{PtcO}_{2}$ se torna dependente de fluxo, oferecendo assim informação quantitativa desse fluxo sanguíneo. A aferição da $\mathrm{PtcO}_{2}$ é contínua e pode dar informação útil sobre a oxigenação tecidual sendo, no entanto, inferior à medida da temperatura nos pés. Ambos os métodos são de fácil realização na beira do leito, minimamente invasivos e de baixo custo. Podem sinalizar precocemente hipoperfusão em situações de falência circulatória aguda, no entanto, são inadequados para avaliação de fluxo no choque com vasodilatação ${ }^{13-15,16}$

\section{QUESTÃO: A MEDIDA DO LACTATO SÉRICO DEVE SER UTILIZADA NA PRÁTICA CLÍNICA?}

Recomendação: Sim. Vários estudos demonstram que a medida seriada mostrou-se superior na avaliação da evolução, em comparação com uma medida isolada. Uma diminuição do lactato sérico de $5 \%$ ou uma depuração de $10 \%$ em 4 a 6 horas sugere que a evolução clínica é favorável ${ }^{17}$.

O lactato sérico deve ser obtido no vaso arterial ou venoso misto porque avalia melhor a mistura das regiões em sofrimento hipóxico. O lactato venoso periférico avalia apenas a região correspondente e não está indicado como monitorização da perfusão global.

Grau B2

Racional: A acidose metabólica freqüentemente surge num paciente com hipóxia tecidual e, em geral, a causa é a acidose láctica. Em condições de hipóxia tecidual, o piruvato como produto final da glicólise anaeróbica não é utilizado no ciclo de Krebs e transforma-se em lactato citosólico, que acaba por circular no plasma.

A hiperlactatemia possui um excelente valor prognóstico em diversas formas de falência circulatória. Consistentemente, diversos estudos em pacientes gravemente sépticos ou em choque séptico mostram que a elevação do lactato sérico está associada a elevadas taxas de disfunção orgânica e mortalidade, com valor prognóstico equivalente ou superior a outras variáveis de oxigenação tecidual ou de disfunção orgânica ${ }^{16,18}$. Em pacientes cirúrgicos estáveis ${ }^{19}$ ou instáveis hemodinamicamente, o lactato sérico avalia adequadamente o prognóstico destes pacientes, tanto quanto a incidência de complicações ou mortalidade. Em traumatizados graves, a elevação do lactato sérico avalia o grau da instabilidade hemodinâmica aguda (hipovole- 
mia) e correlaciona-se com a incidência de complicações pós-reanimação e pós-operatória ${ }^{20}$.

Um estudo não controlado em pacientes traumatizados graves avaliou o uso do lactato sérico elevado para definir as intervenções terapêuticas, sugerindo que o prognóstico poderia ser melhorado. Um outro estudo aleatório com terapêutica dirigida pela normalização da $\mathrm{SvO}_{2}$ e do lactato sérico, em pacientes submetidos à cirurgia cardíaca, mostrou diminuição da morbidade ${ }^{21}$.

A interpretação destes resultados possui algumas limitações. Particularmente nos pacientes sépticos, os altos níveis de lactato podem não ser secundários ao metabolismo anaeróbio e, portanto, não indicariam a presença de hipóxia tecidual. O aumento do lactato nesses pacientes pode ser secundário a uma disfunção do piruvato desidrogenase ou aumento da glicólise aeróbica (relação piruvato/lactato normal). A presença de hepatopatia pode diminuir a sua depuração e manter elevados os níveis de lactato. É recomendável, nestes pacientes, sempre avaliar, de forma seriada, as concentrações séricas do lactato. Finalmente, as concentrações de lactato não fornecem informação confiável sobre a distribuição regional do fluxo sangüíneo e, portanto, sobre a presença de hipóxia regional.

\section{QUESTÃO: A MEDIDA DO EXCESSO DE BASE DEVE SER UTILIZADA NA PRÁTICA CLÍNICA?}

Recomendação: Sim. O excesso de base (EB) pode ser usado como um indicador de hipóxia tecidual em situações agudas de baixo fluxo. Em pacientes traumatizados ou cirúrgicos, durante o período de reanimação, o EB pode discriminar aqueles que ainda não completaram a reanimação. Entretanto, o EB não é um indicador confiável, principalmente, após as primeiras horas de reanimação.

Grau B2

Racional: O excesso de base é a quantidade de base em milimoles necessária para titular um litro de sangue arterial total para um $\mathrm{pH}$ de 7,40 , com a amostra completamente saturada com oxigênio a $37^{\circ} \mathrm{C}$ e a $\mathrm{PaCO}_{2}$ de $40 \mathrm{mmHg}$.

O excesso de base é utilizado como um indicador de hipóxia tecidual (acidose metabólica) e pode fornecer informação semelhante ao lactato em determinadas situações $^{22,23}$. O EB aparece como um indicador prognóstico, particularmente em situações de baixo fluxo (hipovolemia/hemorragia, falência cardíaca). Contudo, o lactato sérico e o déficit de base não estão fixamente ligados. As anormalidades no ambiente ácido-basi- co a partir da administração de base, alterações nos tampões corporais, disfunção renal e anormalidades eletrolíticas têm sido postuladas como causas deste desacoplamento observado em um cenário de reanimação completa (ambiente de UTI) ${ }^{24}$.

\section{QUESTÃO: A MONITORIZAÇÃO DA SvO ${ }_{2}$ E DA SvcO TÊM UTILIDADE NA PRÁTICA CLÍNICA?}

Recomendação: Sim. A monitorização da reanimação hemodinâmica na fase aguda da sepse grave e do choque séptico foi utilizada no trabalho de Rivers e col. ${ }^{25}$ incluída na terapêutica precoce dirigida por objetivos e resultou numa diminuição significativa da mortalidade. Neste trabalho, uma $\mathrm{SvcO}_{2}$ maior que $70 \%$ foi usada como meta terapêutica na reanimação da fase aguda.

A sua utilidade em outras formas de choque tem a mesma base de dados para sua recomendação, mas ainda sem um ensaio clínico aleatório para fundamentá-la. Acredita-se que, até prova em contrário, a mesma meta $\left(\mathrm{SvcO}_{2}>70 \%\right.$ ou $\left.\mathrm{SvO}_{2}>65 \%\right)$ deva ser usada para guiar a reanimação mesmo de outras formas de choque.

A saturação venosa de oxigênio não deve ser usada como meta terapêutica em pacientes críticos fora da fase aguda ou de reanimação hemodinâmica.

Grau B1 na fase aguda da sepse grave ou choque séptico

Grau B2 em outras formas de choque

Racional: A hipóxia tecidual é mais bem definida como a presença de um desequilíbrio entre a demanda por oxigênio e a sua oferta real. A saturação venosa de oxigênio representa equilíbrio entre a demanda $\left(\mathrm{VO}_{2}\right)$ e a oferta de oxigênio $\left(\mathrm{DO}_{2}\right)$ no leito drenado ${ }^{26}$. A saturação venosa mista de oxigênio $\left(\mathrm{SvO}_{2}\right)$ representa esse equilíbrio em toda a circulação sistêmica. Tem sido demonstrado que a saturação venosa central do oxigênio $\left(\mathrm{SvcO}_{2}\right)$, obtida através de um acesso venoso central, é um bom substituto para a $\mathrm{SvO}_{2}$, revelando boa correlação linear com ela e variando igualmente nos distúrbios de $\mathrm{DO}_{2}{ }^{27,28}$.

À medida que a $\mathrm{DO}_{2}$ diminui, os tecidos mantêm o consumo de oxigênio para alcançar a sua demanda através de maior taxa de extração do oxigênio $\left(\mathrm{O}_{2} E R\right)$. Tanto um aumento na $\mathrm{O}_{2}$ ER calculada quanto uma diminuição na saturação venosa de oxigênio é o reflexo disso. Assim, uma diminuição da saturação venosa de oxigênio é um sinal precoce de desequilíbrio da oxigenação tecidual ${ }^{26}$.

Embora não exista nenhum valor seguro para a saturação venosa de oxigênio, tem sido proposto que valores 
acima de $65 \%$ para a $\mathrm{SvO}_{2}$ e $70 \%$ para a $\mathrm{SvcO}_{2}$ geralmente representam um adequado equilíbrio da $\mathrm{DO}_{2} \mathrm{e}$ $\mathrm{VO}_{2}$ sistêmicas.

\section{QUESTÃO: A TONOMETRIA GASOSA GASTRINTES- TINAL E CAPNOMETRIA GASOSA SUBLINGUAL TÊM UTILIDADE NA PRÁTICA CLÍNICA?}

Recomendação: Sim, como indicador prognóstico; Não, como orientador de manobras terapêuticas. Estudos clínicos mostram que a tonometria gastrintestinal tem bom valor preditivo de mortalidade quando os resultados mostram acidose gástrica intramucosa persistente, mas não serve como método orientador de manobras terapêuticas ${ }^{29,30}$. A capnometria sublingual é uma técnica recente e aparentemente promissora, mas ainda necessita de maiores estudos clínicos que dêem suporte à sua utilização rotineira.

Grau B1 para capnometria gástrica

Grau B2 para capnometria sublingual

Racional: O princípio da tonometria gástrica e da capnometria gasosa sublingual baseia-se no conhecimento de que quando há hipoperfusão local, a isquemia causa um aumento na produção de íons $\mathrm{H}^{+}$, aumento da formação de lactato e acúmulo de $\mathrm{CO}_{2}$. $\mathrm{O} \mathrm{CO}_{2}$ difunde-se livremente do tecido gástrico ou sublingual para o tonômetro, permitindo sua medida direta $\left(\mathrm{PgCO}_{2}\right.$ ou $\left.\mathrm{PsICO}_{2}\right)$ e o cálculo do $\mathrm{pH}$ intramucoso (pHi) e do hiato (gap) de $\mathrm{CO}_{2}$ $\left(\mathrm{PgCO}_{2}-\mathrm{PaCO}_{2}\right)$. Dessa forma, o $\mathrm{PgCO}_{2}$ e o hiato de $\mathrm{CO}_{2}$ aumentam e o pHi diminui nas situações de hipóxia local, sendo essas alterações mais intensas em situações de hipofluxo. O hiato de $\mathrm{CO}_{2}$ é mais representativo da hipoperfusão locorregional ${ }^{31}$. A sua obtenção anula a participação do bicarbonato e compensa as variações ventilatórias que se encontram refletidas na $\mathrm{PaCO}_{2}$. No entanto, há necessidade de coleta concomitante de uma amostra de sangue arterial ${ }^{32}$.

Vantagens da tonometria gástrica: Trata-se de uma técnica minimamente invasiva, que permite a detecção precoce de alterações perfusionais locorregionais. É útil também como indicador precoce de risco durante a monitorização no peri e pós-operatório de diversas condições cirúrgicas e em outras condições clínicas, como no uso de fármaco vasoativos no choque ${ }^{33}$. Mostra boa correlação com a gravidade do choque.

Desvantagens da tonometria gástrica: é uma técnica relativamente dispendiosa e dependente do operador. Sofre influência da alimentação gástrica, é dependente do uso de bloqueadores $\mathrm{H}_{2}$ e não reflete a perfusão esplâncnica global, mas apenas aquela relacionada ao estômago.

Vantagens da capnometria sublingual: técnica não-invasiva, simples, de fácil operação e com boa correlação com a tonometria gastrintestinal, com os níveis séricos de lactato e $\mathrm{SvO}_{2}^{34-37}$.

Desvantagens da capnometria sublingual: técnica recente, de alto custo, pouca experiência clínica com o método, risco de infecção (contaminação da solução do capnoprobe) e não avalia a oxigenação. A PsICO só pode ser corretamente interpretada como indicador de hipoperfusão à luz da $\mathrm{PaCO}_{2}$.

\section{QUESTÃO: O GRADIENTE VENO-ARTERIAL DE $\mathrm{CO}_{2}$ TEM UTILIDADE NA PRÁTICA CLÍNICA?}

Recomendação: Sim. Permite uma distinção entre estados de choque de baixo e normo ou alto fluxo, avaliação de gravidade e pode ser usado como orientador de manobras terapêuticas durante a RCR. A variável é de fácil obtenção, caso utilize-se uma amostra venosa central ou o $\mathrm{P}_{\mathrm{ET}} \mathrm{CO}_{2}$. É um indicador qualitativo e semiquantitativo da gravidade do hipofluxo, servindo para orientar manobras terapêuticas na RCR.

Grau B2

Racional: O princípio da utilização do gradiente sistêmico veno-arterial de $\mathrm{CO}_{2}$ baseia-se no conhecimento de que, em situações de baixo fluxo sangüíneo, há um aumento da relação $V A / Q$, fazendo com que o $\mathrm{CO}_{2}$ seja "lavado" do capilar pulmonar, com diminuição relativamente acentuada da $\mathrm{PaCO}_{2}$ em relação à $\mathrm{PvCO}_{2}$. Essa diferença é expressiva apenas nas situações de hipóxia estagnante (baixo fluxo), em contraste com os estados de hipóxia hipóxica ou "citotóxica"38-40.

Vantagens: facilidade de obtenção (gasometria arterial e venosa central/mista); bom indicador de baixo fluxo sistêmico; permite avaliação da eficácia de manobras que aumentem o débito cardíaco, como na RCR.

Desvantagens: método invasivo e que pode ser influenciado por alterações respiratórias e fármacos vasoativos, altera-se de modo significativo apenas em estados de choque com baixo fluxo acentuado, sendo um indicador relativamente tardio.

\section{QUESTÃO: A IMAGEM ORTHOGONAL POLARIZA- TION SPECTRAL IMAGING (OPS) TEM UTILIDADE NA PRÁTICA CLÍNICA?}

Recomendação: No momento, não existem evidências que possam recomendar a aplicação desta técnica. Há necessidade de se definir parâmetros para a utilização, 
avaliar a representatividade da terapêutica na microcirculação regional e sua implicação com a microcirculação sistêmica, assim como com o prognóstico e melhora de desfecho final.

Grau D

Racional: A OPS é uma técnica não-invasiva, simples, que permite visualizar a microcirculação regional de órgãos, à beira do leito, com avaliação semiquantitativa, que consiste em iluminar a área de estudo com uma fonte de luz polarizada que é refletida pelos tecidos e absorvida pela hemoglobina, o que permite conhecer a proporção e o fluxo de pequenos vasos $(<20 \mu \mathrm{m})$; filtros específicos eliminam a luz refletida pela superfície dos tecidos e produzem uma imagem de alto contraste através da luz refletida da microcirculação. Desta forma, as hemácias aparecem escuras e os glóbulos brancos e plaquetas são visíveis, algumas vezes, como corpos refringentes. A parede dos vasos não é visualizada. OPS é particularmente útil para estudar tecidos que possuem uma fina camada epitelial como as superfícies mucosas ${ }^{41,42}$.

Há necessidade de validação clínica; existem erros de técnica, tais como pressão excessiva do cateter, presença de saliva e secreções, movimentos voluntários do paciente e do examinador. Considerar o custo do equipamento. Não avalia a oxigenação. Há necessidade de sedação para diminuir os movimentos do paciente. Existe variabilidade de interpretação dos dados entre os observadores. Ainda com pouca perspectiva de utilidade prática para uso clínico diário, no entanto, alterações microvasculares do fluxo sanguíneo são freqüentemente observadas em pacientes com falência cardíaca e sepse graves, alterações essas mais pronunciadas e persistentes nos pacientes que não sobrevivem e estão relacionadas ao desenvolvimento de falência de múltiplos órgãos. OPS pode vir a ser útil para identificar pacientes com choque séptico com alterações persistentes da microcirculação, nos quais "recrutamento microvascular" possa ser um objetivo terapêutico.

Um trabalho demonstrou que em sepse e choque séptico a diminuição da microcirculação avaliada pelo OPS se correlaciona com gravidade, APACHE II, SOFA e desfecho final ${ }^{43}$.

\section{PAINEL DE ESPECIALISTAS DO CONSENSO BRA- SILEIRO DE MONITORIZAÇÃO E SUPORTE HEMO- DINÂMICO}

Comitê Consultivo: Álvaro Réa-Neto, Professor do Departamento de Clínica Médica da UFPR, Chefe da
UTI adulto do Hospital de Clínicas da UFPR, Diretor do CEPETI - Centro de Estudos e Pesquisa em Terapia Intensiva (Curitiba-PR); Ederlon Rezende, Diretor do Serviço de Terapia Intensiva do Hospital do Servidor Público Estadual (São Paulo-SP); Ciro Leite Mendes, Coordenador da UTI Adulto do Hospital de Emergência e Trauma Senador Humberto Lucena e Hospital Santa Paula, Médico da Rotina da UTI adulto do Hospital Universitário - UFPB (João Pessoa, PB); Cid Marcos David, Presidente do Fundo de Educação e Pesquisa da Associação de Medicina Intensiva Brasileira, Doutor em Ciências Médicas, Professor Adjunto da UFRJ (Rio de Janeiro, RJ). Fernando Suparregui Dias, Professor da Faculdade de Medicina da PUC de Porto Alegre, Chefe da UTI geral do Hospital São Lucas da PUC de Porto Alegre (Porto Alegre, RS); Guilherme Schettino, Doutor em Medicina pela Faculdade de Medicina da USP, Médico Assistente da UTI Respiratória do HC da FMUSP, Médico Coordenador da UTI do Hospital Sírio Libanês (São Paulo-SP); Suzana Margareth Ajeje Lobo, Doutora de Medicina pela Universidade de São Paulo, Professora de clínica médica da Faculdade de Medicina de São José do Rio Preto, Coordenadora do Serviço de Terapia Intensiva do Hospital de Base - FAMERP (São José do Rio Preto-SP).

Painel de Especialistas: Alberto Barros, Médico Coordenador da UTI do Hospital Português de Recife (Recife, PE); Eliézer Silva, Doutor em Medicina, Médico Supervisor CTI Hospital Israelita Albert Einstein (São Paulo, SP); Gilberto Friedman, Editor Chefe da Revista Brasileira de Terapia intensiva; Médico do Hospital de Clínicas de Porto Alegre FAMED-UFRGS (Porto Alegre, RS); José Luiz Gomes do Amaral, Doutor em Medicina, Chefe da Disciplina de Anestesiologia, Dor e Terapia Intensiva da UNIFESP (São Paulo, SP); Marcelo Park, Médico Assistente da Unidade de Terapia Intensiva Disciplina de Emergências Clínicas - HC-FMUSP (São Paulo, SP); Maristela Monachini, Doutora em Medicina, Médica Assistente da UTI do Hospital Sírio Libanês (São Paulo,SP); Mirella Cristine de Oliveira, Médica Coordenadora do Hospital do Trabalhador (Curitiba, PR); Murillo Santucci César Assunção, Médico Assistente da Disciplina de Anestesiologia, Dor e Terapia Intensiva da UNIFESP (São Paulo, SP); Nelson Akamine, Médico Supervisor CTI Hospital Israelita Albert Einstein (São Paulo, SP); Patrícia Veiga C Mello, Médica Coordenadora da Disciplina de Emergência da Universidade Estadual do Piauí, Coordenadora da UTI do Hospital de Terapia Intensiva (Terezina, PI); Renata Andréa Pietro Pereira, Enfermeira Chefe do Serviço de Terapia In- 
tensiva do Hospital do Servidor Público Estadual (São Paulo, SP); Rubens Costa Filho, Médico Coordenador CTI do PROCARDÍACO (Rio de Janeiro, RJ); Sebastião Araújo, Médico, Professor Doutor Assistente Departamento de Cirurgia da FCM-UNICAMP (Campinas, SP); Sérgio Félix Pinto, Médico Chefe da UTI do Hospital Universitário da UFMS (Campo Grande-MS); Sérgio Ferreira, Médico Chefe da UTI do Hospital Universitário Getúlio Vargas da UFAM (Manaus, AM); Simone Mattoso Mitushima, Enfermeira da UTI do Hospital Sírio Libanês (São Paulo, SP); Sydney Agareno, Médico Vice-Coordenador da UTI Geral do Hospital Português de Salvador (Salvador, BA); e Yuzeth Nóbrega de Assis Brilhante, Médica Coordenadora da UTI do Hospital da UNIMED João Pessoa (João Pessoa, PB).

\section{REFERÊNCIAS}

01. Deakin CD, Low JL - Accuracy of the advanced trauma life support guidelines for predicting systolic blood pressure using carotid, femoral, and radial pulses: observational study. BMJ, 2000;321(7262):673-674.

02. Rady MY, Rivers EP, Nowak RM - Resuscitation of the critically ill in the ED: responses of blood pressure, heart rate, shock index, central venous oxygen saturation, and lactate. Am J Emerg Med, 1996;14:218-225.

03. Webb AR - Recognizing hypovolaemia. Minerva Anestesiol, 2001;67:185189.

04. Gogos CA, Lekkou A, Papageorgiou O et al - Clinical prognostic markers in patients with severe sepsis: a prospective analysis of 139 consecutive cases. J Infect, 2003;47:300-306.

05. Levy MM, Fink MP, Marshall JC et al - 2001 SCCM/ESICM/ACCP/ATS/ SIS International Sepsis Definitions Conference. Intensive Care Med, 2003;29:530-538.

06. Boldt J - Clinical review: hemodynamic monitoring in the intensive care unit. Crit Care, 2002;6:52-59.

07. Kaplan LJ, McPartland K, Santora TA et al - Start with a subjective assessment of skin temperature to identify hypoperfusion in intensive care unit patients. J Trauma, 2001;50:620-628.

08. Bailey JM, Levy JH, Kopel MA et al - Relationship between clinical evaluation of peripheral perfusion and global hemodynamics in adults after cardiac surgery. Crit Care Med, 1990;18:1353-1356.

09. Vincent JL, Moraine JJ, van der Linden P - Toe temperature versus transcutaneous oxygen tension monitoring during acute circulatory failure. Intensive Care Med, 1988;14:64-68.

10. Joly HR, Weil MH - Temperature of the great toe as an indication of the severity of shock. Circulation, 1969;39:131-138.

11. Brock L, Skinner JM, Manders JT - Observations on peripheral and central temperatures with particular reference to the occurrence of vasoconstriction. Br J Surg, 1975;62:589-595.

12. Kholoussy $\mathrm{AM}$, Sufian $\mathrm{S}$, Pavlides $\mathrm{C}$ et al - Central peripheral temperature gradient. Its value and limitations in the management of critically ill surgical patients. Am J Surg, 1980;140:609-612.

13. Tremper KK, Barker SJ - Transcutaneous oxygen measurement: experimental studies and adult applications. Int Anesthesiol Clin, 1987;25:67-96.

14. Tatevossian RG, Wo CC, Velmahos GC et al - Transcutaneous oxygen and $\mathrm{CO} 2$ as early warning of tissue hypoxia and hemodynamic shock in critically ill emergency patients. Crit Care Med, 2000;28:2248-2253.

15. Gottrup F, Gellett S, Kirkegaard L et al - Continuous monitoring of tissue oxygen tension during hyperoxia and hypoxia: relation of subcutaneous, transcutaneous, and conjunctival oxygen tension to hemodynamic variables. Crit Care Med, 1988;16:1229-1234.

16. Friedman G, Berlot G, Kahn RJ et al - Combined measurements of blood lactate concentrations and gastric intramucosal $\mathrm{pH}$ in patients with severe sepsis. Crit Care Med, 1995;23:1184-1193.
17. Nguyen HB, Rivers EP, Knoblich BP et al - Early lactate clearance is associated with improved outcome in severe sepsis and septic shock. Crit Care Med, 2004;32:1637-1642.

18. Bakker J, Gris P, Coffernils M et al - Serial blood lactate levels can predict the development of multiple organ failure following septic shock. Am J Surg, 1996;171:221-226

19. Meregalli A, Oliveira RP, Friedman G - Occult hypoperfusion is associated with increased mortality in hemodynamically stable, high-risk, surgical patients. Crit Care, 2004;8:R60-R65.

20. Blow O, Magliore L, Claridge JA et al - The golden hour and the silver day: detection and correction of occult hypoperfusion within 24 hours improves outcome from major trauma. J Trauma, 1999;47:964-969.

21. Polonen $\mathrm{P}$, Ruokonen $\mathrm{E}$, Hippelainen $\mathrm{M}$ et al - A prospective, randomized study of goal-oriented hemodynamic therapy in cardiac surgical patients. Anesth Analg, 2000;90:1052-1059.

22. Smith I, Kumar P, Molloy S et al - Base excess and lactate as prognostic indicators for patients admitted to intensive care. Intensive Care Med, 2001;27:74-83.

23. Husain FA, Martin MJ, Mullenix PS et al - Serum lactate and base deficit as predictors of mortality and morbidity. Am J Surg, 2003;185:485-491.

24. Jeng JC, Jablonski K, Bridgeman A et al - Serum lactate, not base deficit, rapidly predicts survival after major burns. Burns, 2002;28:161-166.

25. Rivers E, Nguyen B, Havstad S et al - Early goal-directed therapy in the treatment of severe sepsis and septic shock. N Engl J Med, 2001;345:13681377.

26. Rivers EP, Ander DS, Powell D - Central venous oxygen saturation monitoring in the critically ill patient. Curr Opin Crit Care, 2001;7:204-211.

27. Reinhart K, Kuhn HJ, Hartog $\mathrm{C}$ et al - Continuous central venous and pulmonary artery oxygen saturation monitoring in the critically ill. Intensive Care Med, 2004;30:1572-1578.

28. Ladakis C, Myrianthefs P, Karabinis A et al - Central venous and mixed venous oxygen saturation in critically ill patients. Respiration, 2001;68:279285.

29. Gutierrez G, Palizas F, Doglio G et al - Gastric intramucosal pH as a therapeutic index of tissue oxygenation in critically ill patients. Lancet, 1992;339(8787):195-199.

30. Gomersall CD, Joynt GM, Freebairn RC et al - Resuscitation of critically ill patients based on the results of gastric tonometry: a prospective, randomized, controlled trial. Crit Care Med, 2000;28:607-614.

31. Neviere R, Chagnon JL, Teboul JL et al - Small intestine intramucosal PCO2 and microvascular blood flow during hypoxic and ischemic hypoxia. Crit Care Med, 2002;30:379-384

32. Maynard N, Bihari D, Beale R et al - Assessment of splanchnic oxygenation by gastric tonometry in patients with acute circulatory failure. JAMA, 1993;270:1203-1210.

33. Doglio GR, Pusajo JF, Egurrola MA et al - Gastric mucosal $\mathrm{pH}$ as a prognostic index of mortality in critically ill patients. Crit Care Med, 1991;19:1037-1040.

34. Marik PE - Sublingual capnography: a clinical validation study. Chest, 2001;120:923-927.

35. Marik PE, Bankov A - Sublingual capnometry versus traditional markers of tissue oxygenation in critically ill patients. Crit Care Med, 2003;31:818-822.

36. Rackow EC, O'Neil P, Astiz ME et al - Sublingual capnometry and indexes of tissue perfusion in patients with circulatory failure. Chest, 2001;120:16331638.

37. Weil MH, Nakagawa $Y$, Tang W et al - Sublingual capnometry: a new noninvasive measurement for diagnosis an quantitation of severity of circulatory shock. Crit Care Med, 1999;27:1225-1229

38. Bowles SA, Schlichtig R, Kramer DJ et al - Arteriovenous $\mathrm{pH}$ and partia pressure of carbon dioxide detect critical oxygen delivery during progressive hemorrhage in dogs. J Crit Care, 1992;7:95-105.

39. Bakker J, Vincent JL, Gris $P$ et al - Veno-arterial carbon dioxide gradient in human septic shock. Chest, 1992;101:509-515.

40. Jin X, Weil MH, Tang $\mathrm{W}$ et al - End-tidal carbon dioxide as a noninvasive indicator of cardiac index during circulatory shock. Crit Care Med, 2000;28:2415-2419.

41. Mathura KR, Vollebregt KC, Boer $\mathrm{K}$ et al - Comparison of OPS imaging and conventional capillary microscopy to study the human microcirculation. J App Physiol, 2001;91:74-78.

42. Ince C - The microcirculation unveiled. Am J Respir Crit Care Med, 2002;166:1-2.

43. De Backer D, Creteur J, Preiser JC et al - Microvascular blood flow is altered in patients with sepsis. Am J Respir Crit Care Med, 2002;166:98-104. 